\title{
A higher frequency of lumbar ossification of the posterior longitudinal ligament in elderly in an outpatient clinic in Japan
}

This article was published in the following Dove Press journal:

International Journal of General Medicine

26 August 2013

Number of times this article has been viewed

\author{
Toshikatsu Okumura' \\ Masumi Ohhira' \\ Shima Kumei' \\ Tsukasa Nozu ${ }^{2}$ \\ 'Department of General Medicine, \\ ${ }^{2}$ Department of Regional Medicine \\ and Education, Asahikawa Medical \\ University, Asahikawa, Japan
}

Purpose: Little is known about the prevalence and epidemiological characteristics of lumbar ossification of the posterior longitudinal ligament (OPLL). We analyzed the rate of lumbar OPLL in an outpatient unit where primary care physicians are working in Japan, to better understand the epidemiological characteristics of the disease.

Methods: We analyzed consecutive, first-time visiting outpatients who received abdominal and pelvic computed tomography (CT) scan at the Department of General Medicine, Asahikawa Medical University Hospital, Japan, between April 2009 and March 2012. Each parameter such as age, sex, and clinical presentation was investigated.

Results: Out of 393 patients who underwent abdominal and pelvic CT scan, 33 (8.4\%) were diagnosed as lumbar OPLL. When compared with patients without lumbar OPLL ( $\mathrm{n}=360)$, there was no significant difference in gender, body mass index (BMI), presence of hypertension, diabetes mellitus or hyperlipidemia, and smoking habit, while the age in patients with lumbar OPLL was significantly higher.

Conclusion: These results suggest for the first time that lumbar OPLL is frequently observed in elderly people in the primary care setting, in Japan.

Keywords: lumbar ossification of the posterior longitudinal ligament, epidemiology, primary care, elderly

\section{Introduction}

Ossification of the posterior longitudinal ligament (OPLL), a result of heterotopic ossification, can induce spinal canal stenosis and lead to the development of severe myelopathy. There have been numerous studies of surgical techniques or clinical outcomes for OPLL of the cervical spine. ${ }^{1}$ In contrast, there are only a few reports regarding OPLL of the lumbar spine, and its clinical characteristics have not yet been well established because the number of patients who require surgical treatment is quite low. ${ }^{2}$ To our knowledge, there has been only six reports of surgically treated lumbar OPLL published in English.? According to the data in the database for the spinal injuries center in Fukuoka, Japan, during the past 27 years, although 6,192 patients underwent operations for degenerative lumbar spine diseases, only ten underwent surgery for lumbar OPLL. ${ }^{2}$ Thus, the frequency of lumbar OPLL requiring surgical treatment was remarkably low, indicating a lack of awareness of the disease even among orthopedic surgeons. It is furthermore speculated that primary care physicians are not familiar with the disease.

We have recently witnessed that radiculopathy due to lumbar OPLL was found to be the cause of chronic right lower abdominal pain in a Japanese man. ${ }^{8}$ After encountering this unusual case, we thought that lumbar OPLL should be considered
Correspondence: Toshikatsu Okumura Department of General Medicine, Asahikawa Medical University,

2-I-I Midorigaoka-Higashi,

Asahikawa 078-8510, Japan

Tel $+8|16668284|$

Fax +81166682846

Email okumurat@asahikawa-med.ac.jp 
to be a factor for not only orthopedic symptoms but also abdominal symptoms. Because abdominal symptoms are common in the primary care setting, ${ }^{9}$ we tried to clarify the incidence of lumbar OPLL in an outpatient clinic in Japan where primary care physicians are working.

\section{Methods}

We analyzed consecutive outpatients making their first visit to the Department of General Medicine, Asahikawa Medical University Hospital, between April 2009 and March 2012. As we have recently demonstrated, ${ }^{9-13}$ the hospital consists of 602 beds in which approximately 250 doctors are working to address almost all medical problems. Among them, four or five primary care physicians are working in the Department of General Medicine. During the period, we analyzed the patients $(n=393)$ who received abdominal and pelvic computed tomography (CT) scan to clarify their several problems such as abdominal symptoms, origins of fever, high level of tumor markers, and so on. Since it has been widely established that $\mathrm{CT}$ is a useful tool for detecting and accurately locating OPLL, ${ }^{14}$ lumbar OPLL was diagnosed by the CT scan according to a previous report. ${ }^{2}$ All data were drawn from medical records and the Computerized Physician Order Entry System in the hospital. Each parameter such as age, sex, body mass index (BMI), and clinical presentation was investigated from the source. Analyzed comorbid conditions included diabetes mellitus, systemic hypertension, dyslipidemia, and cigarette smoking.

\section{Statistical analysis}

Statistical analysis was performed by Fisher's exact test, and Student's $t$-test for age and BMI. A level of $P<0.05$ was considered to be statistically significant.

\section{Results}

Lumbar OPLL was diagnosed by CT according to a previous report. ${ }^{2}$ Representative images are shown in Figure 1. As shown in this figure, OPLL was diagnosed when an apparent ossification of the ligament causing more than $10 \%$ canal stenosis was revealed with CT. Out of 393 patients who underwent abdominal and pelvic CT scan, 33 (8.4\%) were diagnosed with lumbar OPLL. All 33 patients diagnosed with lumbar OPLL detected by CT scan did not complain of main symptoms related to spinal stenosis, such as intermittent claudication. Table 1 shows the clinical characteristics of 33 patients with lumbar OPLL in this study. When compared with patients without lumbar OPLL $(n=360)$, there was no significant difference in gender, BMI, presence of hypertension, diabetes mellitus or hyperlipidemia, and smoking habit, while a significant difference was identified in age in patients with lumbar OPLL. As clearly demonstrated in Table 2, the incidence of lumbar OPLL increased in the elderly. The highest incidence was observed in male patients aged 50-69 years and female patients aged 70-89 years.

Among 33 OPLL patients, seven patients complained of lower abdominal pain as their chief complaint. In the unique case mentioned earlier, the patient's pain was considered to be dependent on lumbar OPLL as reported in our recent publication; ${ }^{8}$ in another case, advanced colon cancer was considered to be the cause of abdominal pain. In the remaining five cases, the pain was diagnosed as functional gastrointestinal disorders after careful examination, but we could not completely exclude the possibility that the abdominal pain in the five patients was related to lumbar OPLL. Just one patient complained of leg pain. In this case, lumbar OPLL might be involved in the symptom, and was followed by consultation with the department of orthopedic surgery in our hospital.
A

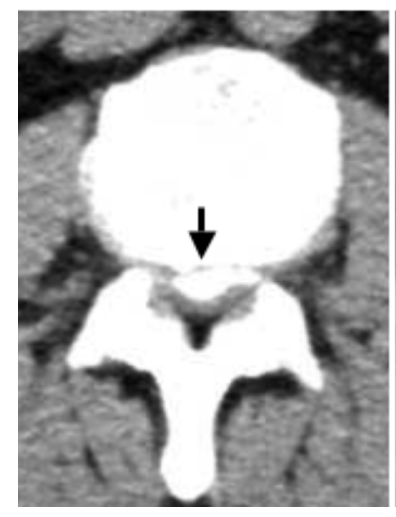

B

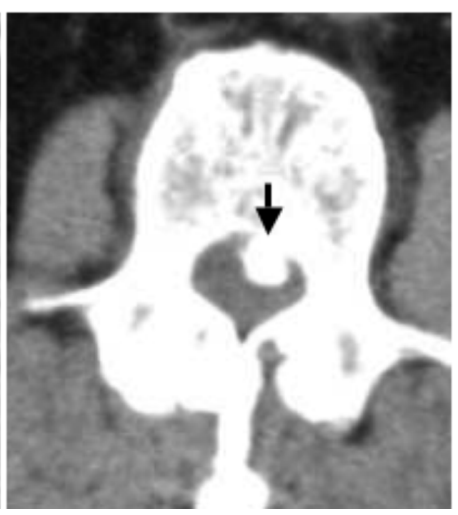

C

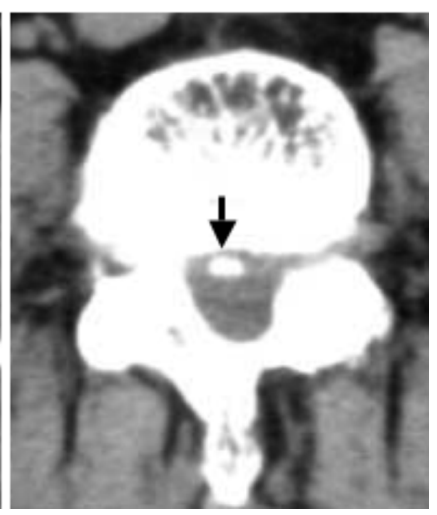

Figure I Abdominal and pelvic CT scans of patients with OPLL.

Notes: Representative images of abdominal and pelvic CT scans in three different cases with lumbar OPLL (A-C). Arrows indicate OPLL.

Abbreviations: OPLL, ossification of the posterior longitudinal ligament; CT, computed tomography. 
Table I Clinical characteristics in patients with lumbar OPLL

\begin{tabular}{lll}
\hline & OPLL (+) & OPLL (-) \\
\hline Gender & & \\
$\quad$ Female (\%) & 57.6 & 51.7 \\
Age (mean) & $64.7^{*}$ & 55.2 \\
BMI (mean) & 23.7 & 22.5 \\
HT (\%) & 30.3 & 18.3 \\
DM (\%) & 12.1 & 9.7 \\
HL (\%) & 12.1 & 12.8 \\
Smoking (\%) & 36.4 & 30.8 \\
\hline
\end{tabular}

Notes: $* P<0.01$, when compared with OPLL (-).

Abbreviations: OPLL, ossification of the posterior longitudinal ligament; BMI, body mass index; $\mathrm{HT}$, hypertension; DM, diabetes mellitus; $\mathrm{HL}$, hyperlipidemia;

\section{Discussion}

OPLL is more common in East Asian populations, particularly in the Japanese, ${ }^{15}$ the prevalence of OPLL is highest in Japan, at a rate of $1.9 \%-4.3 \%{ }^{16}$ Although a number of authors have reported on OPLL of the cervical spine, few have discussed OPLL of the lumber spine. ${ }^{7}$ The number of reports regarding lumbar OPLL has been limited, and its prevalence and regional difference remain unclear. A lack of evidence may be a result of a large majority of lumbar OPLL possibly being asymptomatic, as described below. Clinical presentations in patients with OPLL correspond to the level and magnitude of spinal cord compression. Cervical and thoracic OPLL typically manifests with signs and symptoms of myelopathy, while the lumbar disease usually manifests with signs and symptoms related to spinal stenosis. ${ }^{3,17,18}$ Matsunaga et al ${ }^{17}$ have demonstrated that after 30 years, myelopathy-free rates as high as $71 \%$ have been reported among patients who had no myelopathy when the cervical OPLL was first diagnosed. Although we do not have any evidence that the time-course changes in lumbar OPLL are similar to cervical OPLL, these results led us to speculate that a large majority of lumbar OPLL patients are asymptomatic. In fact, all patients with lumbar OPLL

Table 2 Relationship between age, sex, and lumbar OPLL detected by $\mathrm{CT}$ scan

\begin{tabular}{llllll}
\hline Age & $\mathbf{N}(\mathbf{M} / \mathbf{F})$ & OPLL (M/F) & $\%$ (total) & $\mathbf{M}(\%)$ & $\mathbf{F ~ ( \% )}$ \\
\hline$\leq 19$ & $15(8 / 7)$ & 0 & 0 & 0 & 0 \\
$20-29$ & $25(15 / 10)$ & $\mathrm{I}(0 / 1)$ & 4 & 0 & 10 \\
$30-39$ & $60(30 / 30)$ & $\mathrm{I}(1 / 0)$ & 1.7 & 3 & 0 \\
$40-49$ & $50(24 / 26)$ & $3(1 / 2)$ & 6 & 4.1 & 7.7 \\
$50-59$ & $51(22 / 29)$ & $5(3 / 2)$ & 9.6 & 13.6 & 6.9 \\
$60-69$ & $80(39 / 41)$ & $9(6 / 3)$ & 11.3 & 15.4 & 7.3 \\
$70-79$ & $64(25 / 39)$ & $8(1 / 7)$ & 12.5 & 4 & 17.9 \\
$\geq 80$ & $48(25 / 23)$ & $6(2 / 4)$ & 12.5 & 8 & 17.4 \\
Total & $393(188 / 205)$ & $33(14 / 19)$ & 8.4 & 7.4 & 9.3 \\
\hline
\end{tabular}

Abbreviations: OPLL, ossification of the posterior longitudinal ligament; $\mathrm{CT}$, computed tomography. detected in this study did not complain of spinal stenosismediated symptoms as described above. We do not know at this moment whether lumbar OPLL is indeed rare in the general population, or whether physicians are not aware of the presence of lumbar OPLL. In the present study, out of 393 patients who underwent abdominal and pelvic CT scans, $33(8.4 \%)$ were diagnosed as lumbar OPLL, indicating for the first time the incidence of lumbar OPLL in Japan. According to previous data, the prevalence of cervical and thoracic OPLL in Japanese and East Asian countries has ranged from $1.9 \%-4.3 \% .^{15}$ These results suggest that the frequency of lumbar OPLL is not much lower than that of cervical and thoracic OPLL. ${ }^{1}$

Kobashi et al have demonstrated a higher frequency of diabetes mellitus among Japanese men and women with OPLL, ${ }^{19}$ suggesting that diabetes mellitus may play a role in the development of OPLL. The present results demonstrated that there was no relationship between the presence of diabetes mellitus and lumbar OPLL. The discrepancy may be due to the difference in pathophysiology between cervical and lumbar OPLL. Because of a lack of evidence whether pathogenesis is common in patients with cervical and lumbar OPLL, further studies on this issue are needed. The present study also showed a lack of association of hypertension, hyperlipidemia, and BMI with lumbar OPLL.

\section{Conclusion}

The present study clearly demonstrated for the first time that lumbar OPLL is highly identified in elderly people. Based on these findings, we should pay special attention in elderly patients and work to determine whether they may have lumbar OPLL. Although the presence of lumbar OPLL by itself does not necessarily mean a clinical problem, we should keep in mind that lumbar OPLL is possibly involved in not only spinal stenosis-mediated symptoms, but also radiculopathyrelated symptoms seen in the primary care setting, such as abdominal pain. ${ }^{8}$

\section{Disclosure}

The authors report no conflicts of interest in this work.

\section{References}

1. Schmidt MH, Quinones-Hinojosa A, Rosenberg WS. Cervical myelopathy associated with degenerative spine disease and ossification of the posterior longitudinal ligament. Semin Neurol. 2002;22(2):143-148.

2. Okada S, Maeda T, Saiwai H, Ohkawa Y, Shiba K, Iwamoto Y. Ossification of the posterior longitudinal ligament of the lumbar spine: a case series. Neurosurgery. 2010;67(5):1311-1318; discussion 1318.

3. Kawaguchi Y, Oya T, Abe Y, et al. Spinal stenosis due to ossified lumbar lesions. J Neurosurg Spine. 2005;3(4):262-270. 
4. Epstein N. The surgical management of ossification of the posterior longitudinal ligament in 51 patients. J Spinal Disord. 1993;6(5):432-454; discussion 454-455.

5. Kurihara A, Tanaka Y, Tsumura N, Iwasaki Y. Hyperostotic lumbar spinal stenosis. A review of 12 surgically treated cases with roentgenographic survey of ossification of the yellow ligament at the lumbar spine. Spine. 1988;13(11):1308-1316.

6. Tamura M, Machida M, Aikawa D, et al. Surgical treatment of lumbar ossification of the posterior longitudinal ligament. Report of two cases and description of surgical technique. J Neurosurg Spine. 2005;3(3): 230-233.

7. Liao CC, Lee ST. Symptomatic ossification of the posterior longitudinal ligament of the lumbar spine. Case report. $J$ Neurosurg. 1999;91(Suppl 2):230-232.

8. Okumura T, Ebisawa K, Ohhira M, et al. Chronic right lower abdominal pain due to lumbar ossification of the posterior longitudinal ligament. Am J Gastroenterol. 2012;107(1):142-143.

9. Okumura T, Tanno S, Ohhira M, Tanno S. Prevalence of functional dyspepsia in an outpatient clinic with primary care physicians in Japan. J Gastroenterol. 2010;45(2):187-194.

10. Tanno S, Ohhira M, Tsuchiya Y, Takeuchi T, Tanno S, Okumura T. Frequent early discontinuation of SSRI prescribed by primary care physicians in young males in Japan. Intern Med. 2009;48(15):1263-1266.

11. Okumura T, Tanno S, Ohhira M, Tanno S, Nozu T. Characteristics in patients with headache in an outpatient clinic in Japan. Asia Pac Fam Med. 2010;9(1):10.

12. Okumura T, Tanno S, Ohhira M, Nozu T. The rate of polymyalgia rheumatica (PMR) and remitting seronegative symmetrical synovitis with pitting edema (RS3PE) syndrome in a clinic where primary care physicians are working in Japan. Rheumatol Int. 2012;32(6):1695-1699.
13. Okumura T, Ohhira M, Nozu T. High rate of smoking in female patients with Mondor's disease in an outpatient clinic in Japan. Int J Gen Med. 2012;5:735-738.

14. Saetia K, Cho D, Lee S, Kim DH, Kim SD. Ossification of the posterior longitudinal ligament: a review. Neurosurg Focus. 2011;30(3):E1.

15. Kalb S, Martirosyan NL, Perez-Orribo L, Kalani MY, Theodore N. Analysis of demographics, risk factors, clinical presentation, and surgical treatment modalities for the ossified posterior longitudinal ligament. Neurosurg Focus. 2011;30(3):E11.

16. Stetler WR, La Marca F, Park P. The genetics of ossification of the posterior longitudinal ligament. Neurosurg Focus. 2011;30(3):E7.

17. Matsunaga S, Sakou T, Taketomi E, Komiya S. Clinical course of patients with ossification of the posterior longitudinal ligament: a minimum 10-year cohort study. J Neurosurg. 2004;100(Suppl Spine 3): 245-248.

18. Min JH, Jang JS, Lee SH. Clinical results of ossification of the posterior longitudinal ligament (OPLL) of the thoracic spine treated by anterior decompression. J Spinal Disord Tech. 2008;21(2):116-119.

19. Kobashi G, Washio M, Okamoto K, et al; Japan Collaborative Epidemiological Study Group for Evaluation of Ossification of the Posterior Longitudinal Ligament of the Spine Risk. High body mass index after age 20 and diabetes mellitus are independent risk factors for ossification of the posterior longitudinal ligament of the spine in Japanese subjects: a case-control study in multiple hospitals. Spine. 2004;29(9): 1006-1010.
International Journal of General Medicine

\section{Publish your work in this journal}

The International Journal of General Medicine is an international, peer-reviewed open-access journal that focuses on general and internal medicine, pathogenesis, epidemiology, diagnosis, monitoring and treatment protocols. The journal is characterized by the rapid reporting of reviews, original research and clinical studies across all disease areas.

\section{Dovepress}

A key focus is the elucidation of disease processes and management protocols resulting in improved outcomes for the patient. The manuscript management system is completely online and includes a very quick and fair peer-review system. Visit http://www.dovepress.com/ testimonials.php to read real quotes from published authors. 